\title{
Identifying an unknown time dependent coefficient for quasilinear parabolic equations
}

\author{
Fatma Kanca ${ }^{1}$ and Irem Baglan ${ }^{2}$ \\ ${ }^{1}$ Department of Management Information Systems, Kadir Has University, Istanbul, Turkey \\ ${ }^{2}$ Department of Mathematics, Kocaeli University, Kocaeli, Turkey
}

Received: 6 January 2016, Accepted: 25 January 2016

Published online: 22 June 2016.

\begin{abstract}
This article deals with the mathematical analysis of the inverse problem of identifying the unknown time-dependent coefficient in the quasilinear parabolic equation with the nonlocal boundary and integral overdetermination conditions. The existence, uniqueness and continuously dependence upon the data of the solution are proved by iteration method in addition to the numerical solution of this problem is considered with an example.
\end{abstract}

Keywords: Quasilinear parabolic equation,inverse problem, nonlocal boundary condition, finite difference method, integral overdetermination condition.

\section{Introduction}

Nonlocal boundary conditions such as (4) arise from many important applications in heat transfer, termoelasticity, control theory, life sciences, etc. For example, in heat propagation in a thin rod in which the law of variation $E(t)$ of the total quantity of heat in the rod is given in [4]. In [3] the nature of (3)-type conditions is demonstrated. The problem of identifying of a coefficient are widely for mathematical modeling of various process of physics, chemistry, ecology and industry. For surveys on the subject, we refer the reader to $[1,6,5,7,2]$ and the references therein.

Consider the problem of finding a pair of functions $(r, u)$ satisfying the following quasilinear parabolic problem :

$$
\begin{aligned}
& u_{t}=u_{x x}-p(t) u+r(t) f(x, t, u), \quad(x, t) \in D \\
& u(x, 0)=\varphi(x), \quad x \in[0,1], \\
& u(0, t)=u(1, t), u_{x}(1, t)=0, t \in[0, T], \\
& E(t)=\int_{0}^{1} u(x, t) d x, 0 \leq t \leq T,
\end{aligned}
$$

where

$$
D:=\{0<x<1,0<t<T\}
$$

This problem will be called an inverse problem. The functions $\varphi(x)$ and $f(x, t, u)$ are given functions on $[0,1]$ and $\bar{D} \times(-\infty, \infty)$, respectively. 
The problem of finding the pair $\{r(t), u(x, t)\}$ in (1)-(4) will be called an inverse problem.

In Section 2, the existence and uniqueness of the solution of inverse problem (1)-(4) is proved by using the Fourier method and iteration method. In Section 3, the continuous dependence upon the data of the inverse problem is shown. In Section 4 , the numerical procedure for the solution of the inverse problem is given. In Section 5, some examples are presented.

\section{Existence and uniqueness of the solution of the inverse problem}

Consider the following system of functions on the interval $[0,1]$ :

$$
\begin{aligned}
& X_{0}(x)=2, X_{2 k-1}(x)=4 \cos (2 \pi k x), X_{2 k}(x)=4(1-x) \sin (2 \pi k x), k=1,2, \ldots, \\
& Y_{0}(x)=x, Y_{2 k-1}(x)=x \cos (2 \pi k x), Y_{2 k}(x)=\sin (2 \pi k x), k=1,2, \ldots
\end{aligned}
$$

The systems of these functions arise in [4] for the solution of a nonlocal boundary value problem in heat conduction.

It is easy to verify that the system of function $X_{k}(x)$ and $Y_{k}(x), k=0,1,2, \ldots$ are biorthonormal on $[0,1]$. They are also Riesz bases in $L_{2}[0,1]$ (see [?]).

Definition 1. The pair $\{r(t), u(x, t)\}$ from the class $C[0, T] \times\left(C^{2,1}(D) \cap C^{1,0}(\bar{D})\right)$ for which conditions (1)-(4) are satisfied, is called the classical solution of the inverse problem (1)-(4).

The main result on existence and uniqueness of the solution of the inverse problem (1)-(4) is presented as follows:

We have the following assumptions on the data of the problem (1)-(4).

(A1) $E(t) \in C^{1}[0, T], p(t) \geq 0, r(t) \in C[0, T]$,

(A2) $\varphi(x) \in C^{4}[0,1], \varphi(0)=\varphi(1), \varphi^{\prime}(1)=0, \varphi^{\prime \prime}(0)=\varphi^{\prime \prime}(1)$,

(A3) (1) Let the function $f(x, t, u)$ is continuous with respect to all arguments in $\bar{D} \times(-\infty, \infty)$ and satisfies the following condition

$$
\left|\frac{\partial^{(n)} f(x, t, u)}{\partial x^{n}}-\frac{\partial^{(n)} f(x, t, \tilde{u})}{\partial x^{n}}\right| \leq b(t, x)|u-\tilde{u}|, n=0,1,2,
$$

where $b(x, t) \in L_{2}(D), b(x, t) \geq 0$,

(2) $f(x, t, u) \in C^{4}[0,1], t \varepsilon[0, T],\left.f(x, t, u)\right|_{x=0}=\left.f(x, t, u)\right|_{x=1},\left.f_{x}(x, t, u)\right|_{x=1}=0,\left.f_{x x}(x, t, u)\right|_{x=0}=\left.f_{x x}(x, t, u)\right|_{x=1}$, (3) $\int_{0}^{1} f(x, t, u) d x \neq 0, \forall t \varepsilon[0, T]$. 
By applying the standard procedure of the Fourier method, we obtain the following representation for the solution of (1)-(3) for arbitrary $r(t)$ :

$$
\begin{aligned}
u(x, t) & =\left[\varphi_{0}+\int_{0}^{t} r(\tau) f_{0}(\tau) e^{-\int_{\tau}^{t} p(s) d s} d \tau\right] X_{0}(x) \\
& +\sum_{k=1}^{\infty}\left[\varphi_{2 k} e^{-(2 \pi k)^{2} t-\int_{0}^{t} p(s) d s}+\int_{0}^{t} r(\tau) f_{2 k}(\tau) e^{-(2 \pi k)^{2}(t-\tau)-\int_{\tau}^{t} p(s) d s} d \tau\right] X_{2 k}(x) \\
& +\sum_{k=1}^{\infty}\left[\left(\varphi_{2 k-1}-4 \pi k t \varphi_{2 k}\right) e^{-(2 \pi k)^{2} t-\int_{0}^{t} p(s) d s}\right] X_{2 k-1}(x) \\
& +\sum_{k=1}^{\infty}\left[\int_{0}^{t} r(\tau)\left(f_{2 k-1}(\tau)-4 \pi k(t-\tau) f_{2 k}(\tau)\right) e^{-(2 \pi k)^{2}(t-\tau)-\int_{\tau}^{t} p(s) d s} d \tau\right] X_{2 k-1}(x)
\end{aligned}
$$

where

$$
\varphi_{k}=\int_{0}^{1} \varphi(x) Y_{k}(x) d x, f_{k}(t)=\int_{0}^{1} f(x, t, u) Y_{k}(x) d x, k=0,1,2, \ldots
$$

Let

$$
\begin{aligned}
u_{0}(t) & =\varphi_{0} e^{-\int_{0}^{t} p(s) d s}+\int_{0}^{t} \int_{0}^{1} r(\tau) f(\xi, \tau, u(\xi, \tau)) e^{-\int_{\tau}^{t} p(s) d s} \xi d \xi d \tau \\
u_{2 k}(t) & =\varphi_{2 k} e^{-(2 \pi k)^{2}-\int_{0}^{t} p(s) d s}+\int_{0}^{t} \int_{0}^{1} r(\tau) f(\xi, \tau, u(\xi, \tau)) e^{-(2 \pi k)^{2}-\int_{\tau}^{t} p(s) d s} \sin 2 \pi k \xi d \xi d \tau \\
u_{2 k-1}(t) & =\left(\varphi_{2 k-1}-4 \pi k t \varphi_{2 k}\right) e^{-(2 \pi k)^{2}-\int_{0}^{t} p(s) d s}+\int_{0}^{t} \int_{0}^{1} r(\tau) f(\xi, \tau, u(\xi, \tau)) \xi \cos 2 \pi k \xi e^{-(2 \pi k)^{2}-\int_{\tau}^{t} p(s) d s} d \xi d \tau \\
& -4 \pi k \int_{0}^{t} \int_{0}^{1}(t-\tau) r(\tau) f(\xi, \tau, u(\xi, \tau)) \sin 2 \pi k \xi e^{-(2 \pi k)^{2}-\int_{\tau}^{t} p(s) d s} d \xi d \tau .
\end{aligned}
$$

Under the conditions $\left(\mathrm{A}_{2}\right)$ and $\left(\mathrm{A}_{3}\right)$ - (2) the series (5) and $\sum_{k=1}^{\infty} \frac{\partial}{\partial x}$ converge uniformly in $\bar{D}$ since their majorizing sums are absolutely convergent. Therefore their sums $u(x, t)$ and $u_{x}(x, t)$ are continuous in $\bar{D}$. In addition, the series $\sum_{k=1}^{\infty} \frac{\partial}{\partial t}$ and $\sum_{k=1}^{\infty} \frac{\partial^{2}}{\partial x^{2}}$ are uniformly convergent for $t \geq \varepsilon>0$ ( $\varepsilon$ is an arbitrary positive number). In addition, $u_{t}(x, t)$ is continuous in $\bar{D}$ because the majorizing sum of $\sum_{k=1}^{\infty} \frac{\partial}{\partial t}$ is absolutely convergent under the condition $\left(\mathrm{A}_{2}\right)$ and $\left(\mathrm{A}_{3}\right)-(2)$. Differentiating (4) we obtain

$$
\int_{0}^{1} u_{t}(x, t) d x=E^{\prime}(t), 0 \leq t \leq T
$$


(5) and (6) yield

$$
r(t)=\frac{p(t) E(t)+E^{\prime}(t)+\sum_{k=1}^{\infty} 8 \pi k\left[\varphi_{2 k} e^{-(2 \pi k)^{2} t-\int_{0}^{t} p(s) d s}+\int_{0}^{t} r(\tau) f_{2 k}(\tau) e^{-(2 \pi k)^{2}(t-\tau)-\int_{\tau}^{t} p(s) d s} d \tau\right]}{\int_{0}^{1} f(x, t, u) d x} .
$$

Definition 2. Denote the set

$$
\{u(t)\}=\left\{u_{0}(t), u_{2 k}(t), u_{2 k-1}(t), k=1, \ldots, n\right\},
$$

of continuous on $[0, T]$ functions satisfying the condition

$$
2 \max _{0 \leq t \leq T}\left|u_{0}(t)\right|+4 \sum_{k=1}^{\infty}\left(\max _{0 \leq t \leq T}\left|u_{2 k}(t)\right|+\max _{0 \leq t \leq T}\left|u_{2 k-1}(t)\right|\right)<\infty,
$$

by $\mathbf{B}_{1}$. Let

$$
\|u(t)\|=2 \max _{0 \leq t \leq T}\left|u_{0}(t)\right|+4 \sum_{k=1}^{\infty}\left(\max _{0 \leq t \leq T}\left|u_{2 k}(t)\right|+\max _{0 \leq t \leq T}\left|u_{2 k-1}(t)\right|\right),
$$

be the norm in $\mathbf{B}_{1}$. It can be shown that $\mathbf{B}_{1}$ is the Banach spaces.

Theorem 1. Let assumptions $\left(A_{1}\right)-\left(A_{3}\right)$ be satisfied. Then the inverse problem (1)-(4) has a unique solution in D.

Proof. An iteration for Fourier coefficient of (5) is defined as follows:

$$
\begin{aligned}
u_{0}^{(N+1)}(t) & =u_{0}^{(0)}(t)+\int_{0}^{t} \int_{0}^{1} r^{(N)}(\tau) f\left(\xi, \tau, u^{(N)}(\xi, \tau)\right) e^{-\int_{\tau}^{t} p(s) d s} \xi d \xi d \tau \\
u_{2 k}^{(N+1)}(t) & =u_{2 k}^{(0)}(t)+\int_{0}^{t} \int_{0}^{1} r^{(N)}(\tau) f\left(\xi, \tau, u^{(N)}(\xi, \tau)\right) e^{-(2 \pi k)^{2}-\int_{\tau}^{t} p(s) d s} \sin 2 \pi k \xi d \xi d \tau \\
u_{2 k-1}^{(N+1)}(t) & =u_{2 k-1}^{(0)}(t)+\int_{0}^{t} \int_{0}^{1} r^{(N)}(\tau) f\left(\xi, \tau, u^{(N)}(\xi, \tau)\right) \xi \cos 2 \pi k \xi e^{-(2 \pi k)^{2}-\int_{\tau}^{t} p(s) d s} d \xi d \tau \\
& -4 \pi k \int_{0}^{t} \int_{0}^{1}(t-\tau) r^{(N)}(\tau) f\left(\xi, \tau, u^{(N)}(\xi, \tau)\right) \sin 2 \pi k \xi e^{-(2 \pi k)^{2}-\int_{\tau}^{t} p(s) d s} d \xi d \tau
\end{aligned}
$$

where, $N=0,1,2, \ldots$ An iteration for (7) is defined as:

$$
r^{(N)}(t)=\frac{p(t) E(t)+E^{\prime}(t)+\sum_{k=1}^{\infty} 8 \pi k\left[\varphi_{2 k} e^{-(2 \pi k)^{2} t-\int_{0}^{t} p(s) d s}+\int_{0}^{t} r^{(N)}(\tau) f_{2 k}(\tau) e^{-(2 \pi k)^{2}(t-\tau)-\int_{\tau}^{t} p(s) d s} d \tau\right]}{\int_{0}^{1} f\left(x, t, u^{(N)}\right) d x}
$$

Let

$$
u_{0}^{(0)}(t)=\varphi_{0} e^{-\int_{0}^{t} p(s) d s}, u_{2 k}^{(0)}(t)=\varphi_{2 k} e^{-(2 \pi k)^{2}-\int_{0}^{t} p(s) d s}, u_{2 k-1}^{(0)}(t)=\left(\varphi_{2 k}-4 \pi k t \varphi_{2 k-1}\right) e^{-(2 \pi k)^{2}-\int_{0}^{t} p(s) d s} .
$$


From the conditions of the theorem, we have $u^{(0)}(t) \in \mathbf{B}_{1}$. Let us write $N=0$ in (8).

$$
u_{0}^{(1)}(t)=u_{0}^{(0)}(t)+\int_{0}^{t} \int_{0}^{1} r^{(0)}(\tau) e^{-\int_{\tau}^{t} p(s) d s}\left[f\left(\xi, \tau, u^{(0)}(\xi, \tau)\right)-f(\xi, \tau, 0)\right] \xi d \xi d \tau+\int_{0}^{t} \int_{0}^{1} r^{(0)}(\tau) f(\xi, \tau, 0) e^{-\int_{\tau}^{t} p(s) d s} \xi d \xi d \tau .
$$

Applying the Cauchy inequality, the Lipschitzs condition and taking the maximum of both sides of the last inequality, we have:

$$
2 \underset{0 \leq t \leq T}{2 \max _{0}}\left|u_{0}^{(1)}(t)\right| \leq 2\left|\varphi_{0}\right|+2 \sqrt{T}\|b(x, t)\|_{L_{2}(D)}\left\|u^{(0)}(t)\right\|_{\mathbf{B}_{1}}\left\|r^{(0)}(t)\right\|_{C[0, T]}+2 \sqrt{T}\|f(x, t, 0)\|_{L_{2}(D)} .
$$

and

$$
\begin{aligned}
u_{2 k}^{(1)}(t) & =u_{2 k}^{(0)}(t)+\int_{0}^{t} \int_{0}^{1} r^{(0)}(\tau)\left[f\left(\xi, \tau, u^{(0)}(\xi, \tau)\right)-f(\xi, \tau, 0)\right] \sin 2 \pi k \xi e^{-(2 \pi k)^{2}(t-\tau)-\int_{\tau}^{t} p(s) d s} d \xi d \tau \\
& +\int_{0}^{t} \int_{0}^{1} r^{(0)}(\tau) f(\xi, \tau, 0) \sin 2 \pi k \xi e^{-(2 \pi k)^{2}(t-\tau)-\int_{\tau}^{t} p(s) d s} d \xi d \tau .
\end{aligned}
$$

Applying the Cauchy inequality, the Hölder inequality, the Bessel inequality, the Lipschitz condition and taking maximum of both sides of the last inequality, we obtain:

$$
4 \sum_{k=1}^{\infty} \max _{0 \leq t \leq T}\left|u_{2 k}^{(1)}(t)\right| \leq 4 \sum_{k=1}^{\infty}\left|\varphi_{2 k}\right|+\frac{1}{\sqrt{3}}\|b(x, t)\|_{L_{2}(D)}\left\|u^{(0)}(t)\right\|_{\mathbf{B}_{1}}\left\|r^{(0)}(t)\right\|_{\mathbf{C}[\mathbf{0}, \mathbf{T}]}+\frac{M}{\sqrt{3}}\left\|r^{(0)}(t)\right\|_{\mathbf{C}[\mathbf{0}, \mathbf{T}]} .
$$

Applying the same estimations to $u_{2 k-1}^{(1)}(t)$, we obtain,

$$
\begin{aligned}
4 \sum_{k=1}^{\infty} \max _{0 \leq t \leq T}\left|u_{2 k-1}^{(1)}(t)\right| & \leq 4 \sum_{k=1}^{\infty}\left|\varphi_{2 k-1}\right|+8 T \sum_{k=1}^{\infty}\left|\varphi_{2 k-1}^{\prime}\right|+\left(\frac{1}{\sqrt{3}}+4 \sqrt{2} T\right)\|b(x, t)\|_{L_{2}(D)}\left\|u^{(0)}(t)\right\|_{\mathbf{B}_{1}}\left\|r^{(0)}(t)\right\|_{\mathbf{C}[\mathbf{0}, \mathbf{T}]} \\
& +\left(\frac{1}{\sqrt{3}}+4 \sqrt{2} T\right)\left\|r^{(0)}(t)\right\|_{\mathbf{C}[\mathbf{0}, \mathbf{T}]} M .
\end{aligned}
$$

Finally we have the following inequality:

$$
\begin{aligned}
\left\|u^{(1)}(t)\right\|_{\mathbf{B}_{1}} & =2 \max _{0 \leq t \leq T}\left|u_{0}^{(1)}(t)\right|+4 \sum_{k=1}^{\infty}\left(\max _{0 \leq t \leq T}\left|u_{2 k}^{(1)}(t)\right|+\max _{0 \leq t \leq T}\left|u_{2 k-1}^{(1)}(t)\right|\right) \\
& \leq\|\varphi\|+8 T \sum_{k=1}^{\infty}\left|\varphi_{2 k-1}^{\prime}\right|+\left(2 \sqrt{T}+\frac{2}{\sqrt{3}}+4 \sqrt{2} T\right)\|b(x, t)\|_{L_{2}(D)}\left\|u^{(0)}(t)\right\|_{\mathbf{B}_{1}}\left\|r^{(0)}(t)\right\|_{\mathbf{C}[\mathbf{0}, \mathbf{T}]} \\
& +\left(2 \sqrt{T}+\frac{2}{\sqrt{3}}+4 \sqrt{2} T\right)\left\|r^{(0)}(t)\right\|_{\mathbf{C}[\mathbf{0}, \mathbf{T}]} M .
\end{aligned}
$$


where $\|\varphi\|=2\left|\varphi_{0}\right|+4 \sum_{k=1}^{\infty}\left[\left|\varphi_{2 k}\right|+\left|\varphi_{2 k-1}\right|\right]$. Hence $u^{(1)}(t) \in \mathbf{B}_{1}$. In the same way, for a general value of $N$ we have

$$
\begin{aligned}
\left\|u^{(N)}(t)\right\|_{\mathbf{B}_{1}} & =2 \max _{0 \leq t \leq T}\left|u_{0}^{(N)}(t)\right|+4 \sum_{k=1}^{\infty}\left(\max _{0 \leq t \leq T}\left|u_{2 k}^{(N)}(t)\right|+\max _{0 \leq t \leq T}\left|u_{2 k-1}^{(N)}(t)\right|\right) \\
& \leq\|\varphi\|+8 T \sum_{k=1}^{\infty}\left|\varphi_{2 k-1}^{\prime}\right|+\left(2 \sqrt{T}+\frac{2}{\sqrt{3}}+4 \sqrt{2} T\right)\|b(x, t)\|_{L_{2}(D)}\left\|u^{(N-1)}(t)\right\|_{\mathbf{B}_{1}}\left\|r^{(N-1)}(t)\right\|_{\mathbf{C}[\mathbf{0}, \mathbf{T}]} \\
& +\left(2 \sqrt{T}+\frac{2}{\sqrt{3}}+4 \sqrt{2} T\right)\left\|r^{(N-1)}(t)\right\|_{\mathbf{C}[\mathbf{0}, \mathbf{T}]} M .
\end{aligned}
$$

Since $u^{(N-1)}(t) \in \mathbf{B}_{1}$ and from the conditions of the theorem, we have $u^{(N)}(t) \in \mathbf{B}_{1}$,

$$
\{u(t)\}=\left\{u_{0}(t), u_{2 k}(t), u_{2 k-1}(t), k=1,2, \ldots\right\} \in \mathbf{B}_{1}
$$

Now we prove that the iterations $u^{(N+1)}(t)$ and $r^{(N+1)}(t)$ converge in $\mathbf{B}_{1}$ and $\mathbf{C}[\mathbf{0}, \mathbf{T}]$, respectively, as $N \rightarrow \infty$.

$$
\begin{aligned}
& u^{(1)}(t)-u^{(0)}(t)=2\left(u_{0}^{(1)}(t)-u_{0}^{(0)}(t)\right)+4 \sum_{k=1}^{\infty}\left[\left(u_{2 k}^{(1)}(t)-u_{2 k}^{(0)}(t)\right)+\left(u_{2 k-1}^{(1)}(t)-u_{2 k-1}^{(0)}(t)\right)\right] \\
& =2\left(\int_{0}^{t} \int_{0}^{1} r^{(0)}(\tau)\left[f\left(\xi, \tau, u^{(0)}(\xi, \tau)\right)-f(\xi, \tau, 0)\right] e^{-\int_{\tau}^{t} p(s) d s} \xi d \xi d \tau\right) \\
& +2 \int_{0}^{t} \int_{0}^{1} r^{(0)}(\tau) f(\xi, \tau, 0) \xi d \xi d \tau \\
& +4 \sum_{k=1}^{\infty} \int_{0}^{t} \int_{0}^{1} r^{(0)}(\tau)\left[f\left(\xi, \tau, u^{(0)}(\xi, \tau)\right)-f(\xi, \tau, 0)\right] e^{-(2 \pi k)^{2}(t-\tau)-\int_{\tau}^{t} p(s) d s} \sin 2 \pi k \xi d \xi d \tau \\
& +4 \sum_{k=1}^{\infty} \int_{0}^{t} \int_{0}^{1} r^{(0)}(\tau) f(\xi, \tau, 0) e^{-(2 \pi k)^{2}(t-\tau)-\int_{\tau}^{t} p(s) d s} \sin 2 \pi k \xi d \xi d \tau \\
& +4 \sum_{k=1}^{\infty} \int_{0}^{t} \int_{0}^{1} r^{(0)}(\tau)\left[f\left(\xi, \tau, u^{(0)}(\xi, \tau)\right)-f(\xi, \tau, 0)\right] e^{-(2 \pi k)^{2}(t-\tau)-\int_{\tau}^{t} p(s) d s} \xi \cos 2 \pi k \xi d \xi d \tau \\
& +4 \sum_{k=1}^{\infty} \int_{0}^{t} \int_{0}^{1} r^{(0)}(\tau) f(\xi, \tau, 0) e^{-(2 \pi k)^{2}(t-\tau)-\int_{\tau}^{t} p(s) d s} \xi \cos 2 \pi k \xi d \xi d \tau \\
& -16 \pi \sum_{k=1}^{\infty} k \int_{0}^{t} \int_{0}^{1}(t-\tau) r^{(0)}(\tau)\left[f\left(\xi, \tau, u^{(0)}(\xi, \tau)\right)-f(\xi, \tau, 0)\right] e^{-(2 \pi k)^{2}(t-\tau)-\int_{\tau}^{t} p(s) d s} \sin 2 \pi k \xi d \xi d \tau \\
& +16 \pi \sum_{k=1}^{\infty} k \int_{0}^{t} \int_{0}^{1}(t-\tau) r^{(0)}(\tau) f(\xi, \tau, 0) e^{-(2 \pi k)^{2}(t-\tau)-\int_{\tau}^{t} p(s) d s} \sin 2 \pi k \xi d \xi d \tau
\end{aligned}
$$


Applying Cauchy inequality, Hölder inequality, Lipschitzs condition and Bessel inequality to the last equation, we obtain:

$$
\begin{gathered}
\left\|u^{(1)}(t)-u^{(0)}(t)\right\|_{B_{1}} \leq\left(2 \sqrt{T}+\frac{2}{\sqrt{3}}+4 \sqrt{2} T\right)\|b(x, t)\|_{L_{2}(D)}\left\|u^{(0)}(t)\right\|_{\mathbf{B}_{1}}\left\|r^{(0)}(t)\right\|_{\mathbf{C}[\mathbf{0}, \mathbf{T}]} \\
+\left(2 \sqrt{T}+\frac{2}{\sqrt{3}}+4 \sqrt{2} T\right)\left\|r^{(0)}(t)\right\|_{\mathbf{C}[\mathbf{0}, \mathbf{T}]} M . \\
A=\left(2 \sqrt{T}+\frac{2}{\sqrt{3}}+4 \sqrt{2} T\right)\|b(x, t)\|_{L_{2}(D)}\left\|u^{(0)}(t)\right\|_{\mathbf{B}_{1}}\left\|r^{(0)}(t)\right\|_{\mathbf{C}[\mathbf{0}, \mathbf{T}]}+\left(2 \sqrt{T}+\frac{2}{\sqrt{3}}+4 \sqrt{2} T\right)\left\|r^{(0)}(t)\right\|_{\mathbf{C}[\mathbf{0}, \mathbf{T}]} M .
\end{gathered}
$$

Applying the same estimations we obtain,

$$
\begin{gathered}
\left\|u^{(2)}(t)-u^{(1)}(t)\right\|_{B_{1}} \leq\left(2 \sqrt{T}+\frac{2}{\sqrt{3}}+4 \sqrt{2} T\right)\left\|r^{(1)}(t)\right\|_{\mathbf{C}[\mathbf{0}, \mathbf{T}]}\|b(x, t)\|_{L_{2}(D)}\left\|u^{(1)}-u^{(0)}\right\|_{B_{1}} \\
+\left(2 \sqrt{T}+\frac{2}{\sqrt{3}}+4 \sqrt{2} T\right)\left\|r^{(1)}(t)-r^{(0)}(t)\right\|_{\mathbf{C}[\mathbf{0}, \mathbf{T}]} M . \\
\left\|r^{(1)}-r^{(0)}\right\|_{\mathbf{C}[\mathbf{0}, \mathbf{T}]} \leq \frac{2 \sqrt{2}}{M(1-2 \sqrt{2})}\left\|r^{(1)}(t)\right\|_{\mathbf{C}[\mathbf{0}, \mathbf{T}]}\|b(x, t)\|_{L_{2}(D)}\left\|u^{(1)}-u^{(0)}\right\|_{\mathbf{B}_{1}} . \\
\left\|u^{(2)}(t)-u^{(1)}(t)\right\|_{\mathbf{B}_{1}} \leq\left(2 \sqrt{T}+\frac{2}{\sqrt{3}}+4 \sqrt{2} T+\frac{2 \sqrt{2}}{(1-2 \sqrt{2})}\right)\left\|r^{(1)}(t)\right\|_{\mathbf{C}[\mathbf{0}, \mathbf{T}]}\|b(x, t)\|_{L_{2}(D)} A
\end{gathered}
$$

For same estimations,

$$
\begin{gathered}
\left\|r^{(2)}-r^{(1)}\right\|_{\mathbf{C}[\mathbf{0}, \mathbf{T}]} \leq \frac{2 \sqrt{2}}{M(1-2 \sqrt{2})}\left\|r^{(2)}(t)\right\|_{\mathbf{C}[\mathbf{0}, \mathbf{T}]}\|b(x, t)\|_{L_{2}(D)}\left\|u^{(2)}-u^{(1)}\right\|_{\mathbf{B}_{1}} \cdot \\
\left\|u^{(3)}(t)-u^{(2)}(t)\right\|_{\mathbf{B}_{1}} \leq \frac{A}{\sqrt{2}}\left(2 \sqrt{T}+\frac{2}{\sqrt{3}}+4 \sqrt{2} T+\frac{2 \sqrt{2}}{(1-2 \sqrt{2})}\right)^{2} x\left\|r^{(1)}(t)\right\|_{\mathbf{C}[\mathbf{0}, \mathbf{T}]}\left\|r^{(2)}(t)\right\|_{\mathbf{C}[\mathbf{0}, \mathbf{T}]}\|b(x, t)\|_{L_{2}\left(D_{T}\right)}^{2} .
\end{gathered}
$$

For $N$ :

$$
\begin{aligned}
& \left\|r^{(N+1)}-r^{(N)}\right\|_{\mathbf{C}[\mathbf{0}, \mathbf{T}]} \leq \frac{2 \sqrt{2}}{M(1-2 \sqrt{2})}\left\|r^{(N+1)}(t)\right\|_{\mathbf{C}[\mathbf{0}, \mathbf{T}]}\|b(x, t)\|_{L_{2}(D)}\left\|u^{(N+1)}-u^{(N)}\right\|_{\mathbf{B}_{1}} . \\
& \left\|u^{(N+1)}(t)-u^{(N)}(t)\right\|_{\mathbf{B}_{1}} \leq \frac{A}{\sqrt{N !}}\left\|r^{(1)}(t)\right\|_{\mathbf{C}[\mathbf{0}, \mathbf{T}]}\left\|r^{(2)}(t)\right\|_{\mathbf{C}[\mathbf{0}, \mathbf{T}]} \ldots\left\|r^{(N)}(t)\right\|_{\mathbf{C}[\mathbf{0}, \mathbf{T}]} x \\
& \left(2 \sqrt{T}+\frac{2}{\sqrt{3}}+4 \sqrt{2} T+\frac{2 \sqrt{2}}{(1-2 \sqrt{2})}\right)^{N}\|b(x, t)\|_{L_{2}\left(D_{T}\right)}^{N} .
\end{aligned}
$$

It is easy to see that if $u^{(N+1)} \rightarrow u^{(N)}, N \rightarrow \infty$, then $r^{(N+1)} \rightarrow r^{(N)}, N \rightarrow \infty$.

Therefore $u^{(N+1)}(t)$ and $r^{(N+1)}(t)$ converge in $\mathbf{B}_{1}$ and $\mathbf{C}[\mathbf{0}, \mathbf{T}]$, respectively. 
Now let us show that there exists $u$ and $r$ such that

$$
\lim _{N \rightarrow \infty} u^{(N+1)}(t)=u(t), \lim _{N \rightarrow \infty} r^{(N+1)}(t)=r(t)
$$

Applying the Cauchy inequality, the Hölder Inequality, the Lipschitzs condition and the Bessel inequality to $\left|u-u^{(N+1)}\right|$ and $\left|r-r^{(N)}\right|$

$$
\begin{aligned}
&\left|r-r^{(N)}\right| \leq \frac{2 \sqrt{2}}{M(1-2 \sqrt{2})}|r(t)||b(x, t)|\left|u-u^{(N+1)}\right| \frac{2 \sqrt{2}}{M(1-2 \sqrt{2})}|r(t)||b(x, t)|\left|u^{(N+1)}-u^{(N)}\right| . \\
&\left|u(t)-u^{(N+1)}(t)\right| \leq\left(2 \sqrt{T}+\frac{2}{\sqrt{3}}+4 \sqrt{2} T+\frac{2 \sqrt{2}}{(1-2 \sqrt{2})}\right) x|r(t)||b(x, t)| \mid u-u^{(N+1) \mid} \\
&+\left(2 \sqrt{T}+\frac{2}{\sqrt{3}}+4 \sqrt{2} T+\frac{2 \sqrt{2}}{(1-2 \sqrt{2})}\right) x|r(t)||b(x, t)|\left|u^{(N+1)}-u^{(N)}\right| .
\end{aligned}
$$

Applying the Gronwall inequality to the last inequality ,using inequality (9) and taking maximum of both sides, we have

$$
\begin{aligned}
\left\|u(t)-u^{(N+1)}(t)\right\|_{B_{1}}^{2} & \leq 2 \frac{A^{2}}{N !}\left(2 \sqrt{T}+\frac{2}{\sqrt{3}}+4 \sqrt{2} T+\frac{2 \sqrt{2}}{(1-2 \sqrt{2})}\right)^{2(N+1)} \\
& \times\left\{\|b(x, t)\|_{L_{2}(D)}^{N+1}\|r(t)\|_{\mathbf{C}[\mathbf{0}, \mathbf{T}]}\left\|r^{(1)}(t)\right\|_{\mathbf{C}[\mathbf{0}, \mathbf{T}]}\left\|r^{(2)}(t)\right\|_{\mathbf{C}[\mathbf{0}, \mathbf{T}]} \ldots\left\|r^{(N)}(t)\right\|_{\mathbf{C}[\mathbf{0}, \mathbf{T}]}\right\}^{2} \\
& \times \exp 2\left(2 \sqrt{T}+\frac{2}{\sqrt{3}}+4 \sqrt{2} T+\frac{2 \sqrt{2}}{(1-2 \sqrt{2})}\right)^{2}\|b(x, t)\|_{L_{2}(D)}^{2}\|r(t)\|_{\mathbf{C}[\mathbf{0}, \mathbf{T}]}^{2}
\end{aligned}
$$

Then $N \rightarrow \infty$ we obtain $u^{(N+1)} \rightarrow u, r^{(N+1)} \rightarrow r$.

For the uniqueness, we assume that problem (1)-(4) has two solution pairs $(r, u),(q, v)$. Applying the Cauchy inequality, the Hölder Inequality, the Lipshitzs condition and the Bessel inequality to $|u(t)-v(t)|$ and $|r(t)-q(t)|$, we obtain:

$$
\begin{gathered}
|u(t)-v(t)| \leq\left(2 \sqrt{T}+\frac{2}{\sqrt{3}}+4 \sqrt{2} T\right)|b(x, t)||r(t)||u(t)-v(t)|+\left(2 \sqrt{T}+\frac{2}{\sqrt{3}}+4 \sqrt{2} T\right)|r(t)-q(t)| M \\
|r(t)-q(t)| \leq \frac{2 \sqrt{2}}{M(1-2 \sqrt{2})}|r(t)||u(t)-v(t)||b(x, t)|,
\end{gathered}
$$

applying the Gronwall inequality to the last inequality, we have $u(t)=v(t)$. Hence $r(t)=q(t)$.

The theorem is proved.

\section{Continuous dependence of $(a, u)$ upon the data}

Theorem 2. Under assumption (A1)-(A3) the solution $(r, u)$ of the problem (1)-(4) depends continuously upon the data $\varphi, E$. 
Proof. Let $\Phi=\{\varphi, E, f\}$ and $\bar{\Phi}=\{\bar{\varphi}, \bar{E}, f\}$ be two sets of the data, which satisfy the assumptions $\left(A_{1}\right)-\left(A_{3}\right)$.Suppose that there exist positive constants $M_{i}, i=1,2$ such that

$$
\|E\|_{C^{1}[0, T]} \leq M_{1},\|\bar{E}\|_{C^{1}[0, T]} \leq M_{1},\|\varphi\|_{C^{3}[0,1]} \leq M_{2},\|\bar{\varphi}\|_{C^{3}[0,1]} \leq M_{2}
$$

Let us denote $\|\Phi\|=\left(\|E\|_{C^{1}[0, T]}+\|\varphi\|_{C^{3}[0,1]}+\|f\|_{C^{3,0}(\bar{D})}\right)$. Let $(r, u)$ and $(\bar{r}, \bar{u})$ be solutions of inverse problems (1)-(4) corresponding to the data $\Phi=\{\varphi, E, f\}$ and $\bar{\Phi}=\{\bar{\varphi}, \bar{E}, f\}$ respectively. According to (5)

$$
\begin{aligned}
& u- \bar{u} \\
&= 2\left(\varphi_{0}-\overline{\varphi_{0}}\right) e^{-\int_{0}^{t} p(s) d s}+4 \sum_{k=1}^{\infty} \sin 2 \pi k \xi\left(\varphi_{2 k}-\overline{\varphi_{2 k}}\right) e^{-(2 \pi k)^{2} t-\int_{0}^{t} p(s) d s} \\
&+4 \sum_{k=1}^{\infty} \cos 2 k \xi\left(\varphi_{2 k-1}-\overline{\varphi_{2 k-1}}\right) e^{-(2 \pi k)^{2} t-\int_{0}^{t} p(s) d s} \\
&+2\left(\int_{0}^{t} \int_{0}^{1} r(\tau)[f(\xi, \tau, u(\xi, \tau))-f(\xi, \tau, \bar{u}(\xi, \tau))] e^{-\int_{\tau}^{t} p(s) d s} d \xi d \tau\right) \\
&+2\left(\int_{0}^{t} \int_{0}^{1}(r(\tau)-\bar{r}(\tau)) f(\xi, \tau, \bar{u}(\xi, \tau)) e^{-\int_{\tau}^{t} p(s) d s} d \xi d \tau\right) \\
&+4 \sum_{k=1}^{\infty} \int_{0}^{t} \int_{0}^{1} r(\tau)[f(\xi, \tau, u(\xi, \tau))-f(\xi, \tau, \bar{u}(\xi, \tau))] \sin 2 \pi k \xi e^{-(2 \pi k)^{2}(t-\tau)-\int_{\tau}^{t} p(s) d s} d \xi d \tau \\
&+4 \sum_{k=1}^{\infty} \int_{0}^{t} \int_{0}^{1}(r(\tau)-\bar{r}(\tau))[f(\xi, \tau, u(\xi, \tau))-f(\xi, \tau, \bar{u}(\xi, \tau))] \sin 2 \pi k \xi e^{-(2 \pi k)^{2}(t-\tau)-\int_{\tau}^{t} p(s) d s} d \xi d \tau \\
&+4 \sum_{k=1}^{\infty} \int_{0}^{t} \int_{0}^{1} r(\tau)[f(\xi, \tau, u(\xi, \tau))-f(\xi, \tau, \bar{u}(\xi, \tau))] \xi \cos 2 \pi k \xi e^{-(2 \pi k)^{2}(t-\tau)-\int_{\tau}^{t} p(s) d s} d \xi d \tau \\
&+4 \sum_{k=1}^{\infty} \int_{0}^{t} \int_{0}^{1}(r(\tau)-\bar{r}(\tau))[f(\xi, \tau, u(\xi, \tau))-f(\xi, \tau, \bar{u}(\xi, \tau))] \xi \cos 2 \pi k \xi e^{-(2 \pi k)^{2}(t-\tau)-\int_{\tau}^{t} p(s) d s} d \xi d \tau \\
&-16 \pi \sum_{k=1}^{\infty} k \int_{0}^{t} \int_{0}^{1}(t-\tau) r(\tau)[f(\xi, \tau, u(\xi, \tau))-f(\xi, \tau, \bar{u}(\xi, \tau))] \sin 2 \pi k \xi e^{-(2 \pi k)^{2}(t-\tau)-\int_{\tau}^{t} p(s) d s} d \xi d \tau \\
&-16 \pi \sum_{k=1}^{\infty} k \int_{0}^{t} \int_{0}^{1}(t-\tau)(r(\tau)-\bar{r}(\tau))[f(\xi, \tau, u(\xi, \tau))-f(\xi, \tau, \bar{u}(\xi, \tau))] \sin 2 \pi k \xi e^{-(2 \pi k)^{2}(t-\tau)-\int_{\tau}^{t} p(s) d s} d \xi d \tau \\
&
\end{aligned}
$$

Same estimations,

$$
\begin{gathered}
|u-\bar{u}| \leq M_{4}\|\Phi-\bar{\Phi}\|+M_{3}\left(\int_{0}^{t} \int_{0}^{\pi} r^{2}(\tau) b^{2}(\xi, \tau)|u(\tau)-\bar{u}(\tau)|^{2} d \xi d \tau\right)^{\frac{1}{2}}, \\
|r-\bar{r}| \leq M_{5}\|\Phi-\bar{\Phi}\|+M_{6}|r(t)||u(t)-\overline{u(t)}|
\end{gathered}
$$

applying Gronwall's inequality to (14), we obtain: 


$$
|u-\bar{u}|^{2} \leq 2 M_{4}^{2}\|\Phi-\bar{\Phi}\|^{2} x \exp 2 M_{3}^{2}\left(\int_{0}^{t} \int_{0}^{\pi} r^{2}(\tau) b^{2}(\xi, \tau) d \xi d \tau\right) .
$$

For $\Phi \rightarrow \bar{\Phi}$ then $u \rightarrow \bar{u}$. Hence $r \rightarrow \bar{r}$.

\section{Numerical procedure for the nonlinear problem (1)-(4)}

We construct an iteration algorithm for the linearization of the problem (1)-(4):

$$
\begin{aligned}
\frac{\partial u^{(n)}}{\partial t} & =\frac{\partial^{2} u^{(n)}}{\partial x^{2}}-p(t) u^{(n)}+r(t) f\left(x, t, u^{(n-1)}\right),(x, t) \in D \\
u^{(n)}(0, t) & =u^{(n)}(1, t), t \in[0, T] \\
u_{x}^{(n)}(1, t) & =0, t \in[0, T] \\
u^{(n)}(x, 0) & =\varphi(x), \quad x \in[0,1] .
\end{aligned}
$$

Let $u^{(n)}(x, t)=v(x, t)$ and $f\left(x, t, u^{(n-1)}\right)=\widetilde{f}(x, t)$. Then the problem (15)-(18) can be written as a linear problem:

$$
\begin{aligned}
\frac{\partial v}{\partial t} & =\frac{\partial^{2} v}{\partial x^{2}}-p(t) v(x, t)+r(t) \widetilde{f}(x, t) \quad(x, t) \in D \\
v(0, t) & =v(1, t), \quad t \in[0, T], \\
v_{x}(1, t) & =0, \quad t \in[0, T], \\
v(x, 0) & =\varphi(x), \quad x \in[0,1] .
\end{aligned}
$$

In [?,?], the problems are linear. In this problem in order to use the similar methods in these papers, firstly we use the method of the linearization then we use the finite difference method to solve (19)-(22) with a predictor-corrector type approach.

We subdivide the intervals $[0,1]$ and $[0, T]$ into $N_{x}$ and $N_{t}$ subintervals of equal lengths $h=\frac{1}{N_{x}}$ and $\tau=\frac{T}{N_{t}}$, respectively. We choose the Crank-Nicolson scheme, which is absolutely stable and has a second order accuracy in both $h$ and $\tau$. The Crank-Nicolson scheme for (19)-(22) is as follows:

$$
\begin{gathered}
\frac{1}{\tau}\left(v_{i}^{j+1}-v_{i}^{j}\right)=\frac{1}{2 h^{2}}\left[\left(v_{i-1}^{j}-2 v_{i}^{j}+v_{i+1}^{j}\right)+\left(v_{i-1}^{j+1}-2 v_{i}^{j+1}+v_{i+1}^{j+1}\right)\right] \\
-\frac{1}{4}\left(p^{j+1}+p^{j}\right)\left(v_{i}^{j+1}+v_{i}^{j}\right)+\frac{1}{4}\left(r^{j}+r^{j+1}\right)\left(\widetilde{f}_{i}^{j+1}+\widetilde{f}_{i}^{j}\right), \\
v_{i}^{0}=\phi_{i}, \\
v_{0}^{j}=v_{N_{x}}^{j},
\end{gathered}
$$




$$
v_{N_{x}-1}^{j}=v_{N_{x}+1}^{j},
$$

where $1 \leq i \leq N_{x}$ and $0 \leq j \leq N_{t}$ are the indices for the spatial and time steps respectively, $v_{i}^{j}=v\left(x_{i}, t_{j}\right), \phi_{i}=\varphi\left(x_{i}\right)$, $\widetilde{f}_{i}^{j}=\widetilde{f}\left(x_{i}, t_{j}\right), p^{j}=p\left(t_{j}\right), x_{i}=i h, t_{j}=j \tau$. At the $t=0$ level, adjustment should be made according to the initial condition and the compatibility requirements.

Now, let us construct the predicting-correcting mechanism. First, integrating the equation (1) respect to $x$ from 0 to 1 and using (3) and (4), we obtain

$$
r(t)=\frac{E^{\prime}(t)+p(t) E(t)+v_{x}(0, t)}{\int_{0}^{1} \widetilde{f}(x, t) d x} .
$$

The finite difference approximation of (27) is

$$
r^{j}=\frac{\left(\left(E^{j+1}-E^{j}\right) / \tau\right)+p^{j} E^{j}+\left(v_{N_{x}+1}^{j}-v_{N_{x}}^{j}\right) / h}{(\widetilde{f} i n)^{j}},
$$

where $E^{j}=E\left(t_{j}\right),(\widetilde{f} \text { in })^{j}=\int_{0}^{1} \widetilde{f}\left(x, t_{j}\right) d x, j=0,1, \ldots, N_{t}$. For $j=0$,

$$
r^{0}=\frac{-\left(\left(E^{1}-E^{0}\right) / \tau\right)+p^{0} E^{0}+\left(\phi_{N_{x}+1}-\phi_{N_{x}}\right) / h}{(\widetilde{\text { fin }})^{0}},
$$

and the values of $\phi_{i}$ provide us to start our computation. We denote the values of $p^{j}, v_{i}^{j}$ at the $s$-th iteration step $r^{j(s)}$, $v_{i}^{j(s)}$, respectively. In numerical computation, since the time step is very small, we can take $r^{j+1(0)}=r^{j}, v_{i}^{j+1(0)}=v_{i}^{j}$, $j=0,1,2, \ldots . N_{t}, i=1,2, \ldots, N_{x}$. At each $(s+1)$-th iteration step we first determine $r^{j+1(s+1)}$ from the formula

$$
r^{j+1(s+1)}=\frac{-\left(\left(E^{j+2}-E^{j+1}\right) / \tau\right)+p^{j+1} E^{j+1}+\left(v_{N_{x}+1}^{j+1(s)}-v_{N_{x}}^{j+1(s)}\right) / h}{(\widetilde{f} i)^{j+1}} .
$$

Then from (23)-(26) we obtain

$$
\begin{gathered}
\frac{1}{\tau}\left(v_{i}^{j+1(s+1)}-v_{i}^{j+1(s)}\right)=\frac{1}{2 h^{2}}\left[\left(v_{i-1}^{j+1(s+1)}-2 v_{i}^{j+1(s+1)}+v_{i+1}^{j+1(s+1)}\right)+\left(v_{i-1}^{j+1(s)}-2 v_{i}^{j+1(s)}+v_{i+1}^{j+1(s)}\right)\right] \\
-\frac{1}{4}\left(p^{j+1}+p^{j}\right)\left(v_{i}^{j+1(s+1)}+v_{i}^{j+1(s)}\right)+\frac{1}{4}\left(r^{j+1(s+1)}+r^{j+1(s)}\right)\left(\widetilde{f}_{i}^{j+1}+\widetilde{f}_{i}^{j}\right), \\
v_{0}^{j+1(s)}=v_{N_{x}+1}^{j+1(s)}, \\
v_{N_{x}-1}^{j(s)}=v_{N_{x}+1}^{j(s)},
\end{gathered}
$$

The system of equations (28)-(30) can be solved by the Gauss elimination method and $v_{i}^{j+1(s+1)}$ is determined. If the difference of values between two iterations reaches the prescribed tolerance, the iteration is stopped and we accept the corresponding values $r^{j+1(s+1)}, v_{i}^{j+1(s+1)}\left(i=1,2, \ldots, N_{x}\right)$ as $r^{j+1}, v_{i}^{j+1}\left(i=1,2, \ldots, N_{x}\right)$, on the $(j+1)$-th time step, respectively. In virtue of this iteration, we can move from level $j$ to level $j+1$. 


\section{Numerical example}

Example 1. If we consider the inverse problem (1)-(4), with

$$
\begin{aligned}
f(x, t, u) & =(2 \pi)^{2} \cos (2 \pi x) \exp (-t)+u(1+\exp (t)) \exp (-t), \\
p(t) & =\exp (t), \varphi(x)=\cos (2 \pi x)+1 \\
E(t) & =\exp (t), \quad x \in[0,1], t \in[0, T]
\end{aligned}
$$

It is easy to check that the analytical solution of this problem is $\{r(t), u(x, t)\}=\{\exp (2 t),(\cos (2 \pi x)+1)) \exp (t)\}$.

Let us apply the scheme which was explained in the previous section for the step sizes $h=0.005, \tau=0.005$.In the case when $T=1$ the comparisons between the analytical solution and the numerical finite difference solution are shown in Figures 1 and 2.

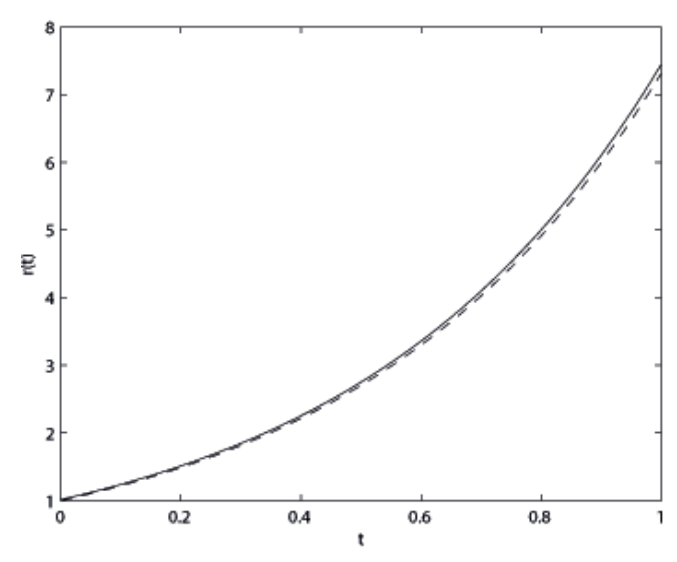

Fig. 1: Exact and approximate $r(t)$ when $T=1$.

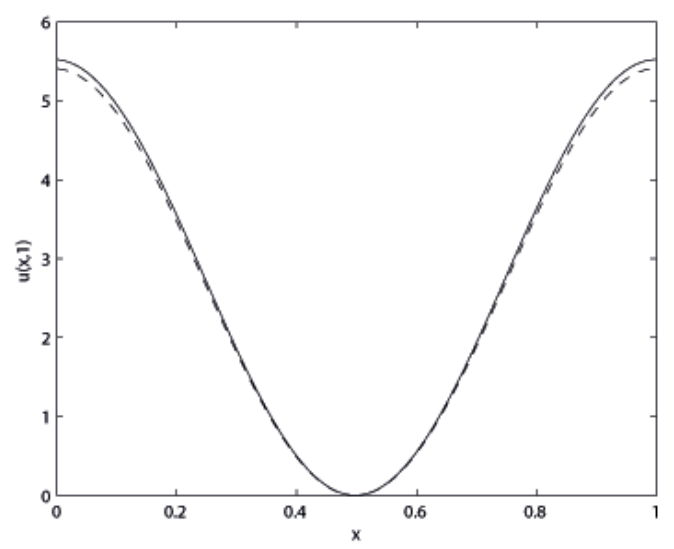

Fig. 2: Exact and approximate solutions of $\mathrm{u}(\mathrm{x}, \mathrm{t})$ at the $\mathrm{T}=1$. 
It is clear from these results, this method has been shown to produce stable and reasonably accurate results for this example.

\section{References}

[1] Kanca F.,Baglan I., Continuous dependence on data for a solution of the quasilinear parabolic equation with a periodic boundary condition, Boundary Value Problems, 28, 2013.

[2] Sakınc I., Numerical Solution of a Quasilinear Parabolic Problem with Periodic Boundary Condition, Hacettepe Journal of Mathematics and Statistics, 2010;39(2):183-189.

[3] A. M. Nakhushev, Equations of Mathematical Biology, Moscow, 1995 (in Russian).

[4] Ionkin NI. Solution of a boundary-value problem in heat conduction with a nonclassical boundary condition. Differential Equations. 1977; 13: 204-211

[5] Pourgholia R, Rostamiana M and Emamjome M., A numerical method for solving a nonlinear inverse parabolic problem. Inverse Problems in Science and Engineering, 2010, 18(8):1151-1164.

[6] Cannon J,R., Lin Y., Determination of parameter p(t) in Hölder classes for some semilinear parabolic equations. Inverse Problems, 1988, 4:595-606.

[7] Ozbilge E., Demir A., Inverse problem for a time-fractional parabolic equation, Journal of Inequalities and Applications, 2015, 81, (Mar 2015). 